Geopolítica(s) Revista de estudios sobre espacio y poder ISSN: 2172-3958

https://dx.doi.org/10.5209/geop.69125

\title{
Editorial. Diez años de Geopolítica(s)
}

Consejo de Redacción

Cómo citar: Consejo de Redacción. (2020). Editorial. Diez años de Geopolítica(s). Geopolítica(s). Revista de estudios sobre espacio y poder, 11(1), 11-20.

Hace diez años salimos "a la calle" — en sentido metafórico, como los periódicos de épocas pasadas, pero ahora en internet- con un objetivo que resumíamos al final de nuestra primera editorial:

En definitiva, Geopolítica(s) quiere contribuir al desarrollo de una perspectiva de análisis plural —en lo metodológico y en lo teórico- y multiescalar sobre las relaciones entre espacio y poder, preferentemente en los países ibéricos y latinoamericanos, pero abiertos al mundo y a su conocimiento global. (Editorial, 2010, p.13).

Este cumpleaños es un buen momento para hacer balance de hasta qué punto se han cubierto esos objetivos $\mathrm{y}$, en general, qué hemos hecho estos diez años pasados. En este sentido, vamos a ocuparnos primero de los artículos publicados y sus temáticas, después de los autores/as (su género, procedencia e idioma utilizado en la publicación), para posteriormente analizar dos secciones de la revista que comenzamos más tarde, pero que han sido muy importantes: los clásicos y los ensayos bibliográficos.

\section{El diagnóstico}

Hemos publicado un total de 110 artículos en estos diez años, lo que supone una media de 5,5 artículos por número, y un esfuerzo importante para una revista que nace hace sólo diez años. Los primeros años no fueron fáciles porque el flujo de artículos que llegaban era muy bajo y a veces había que esperar a finales del plazo de publicación para completar el número. Aún así no se relajó nunca el proceso de evaluación por pares doble ciego, al que se someten los artículos tras ser revisados inicialmente por el Consejo de Redacción. La consolidación de la revista y posiblemente la inclusión en algunos índices de referencia ha hecho que lleguen más artículos, de modo que es más fácil cumplir con las fechas de publicación porque se tiene 
una cartera de artículos para cada número. En cualquier caso, creemos que este conjunto de trabajos, junto con los las secciones de clásicos y ensayos bibliográficos, constituyen una aportación relevante al conocimiento geopolítico, especialmente en el ámbito ibérico y latinoamericano.

Figura 1. Líneas temáticas de los artículos publicados en Geopolítica(s) (2010-2019)

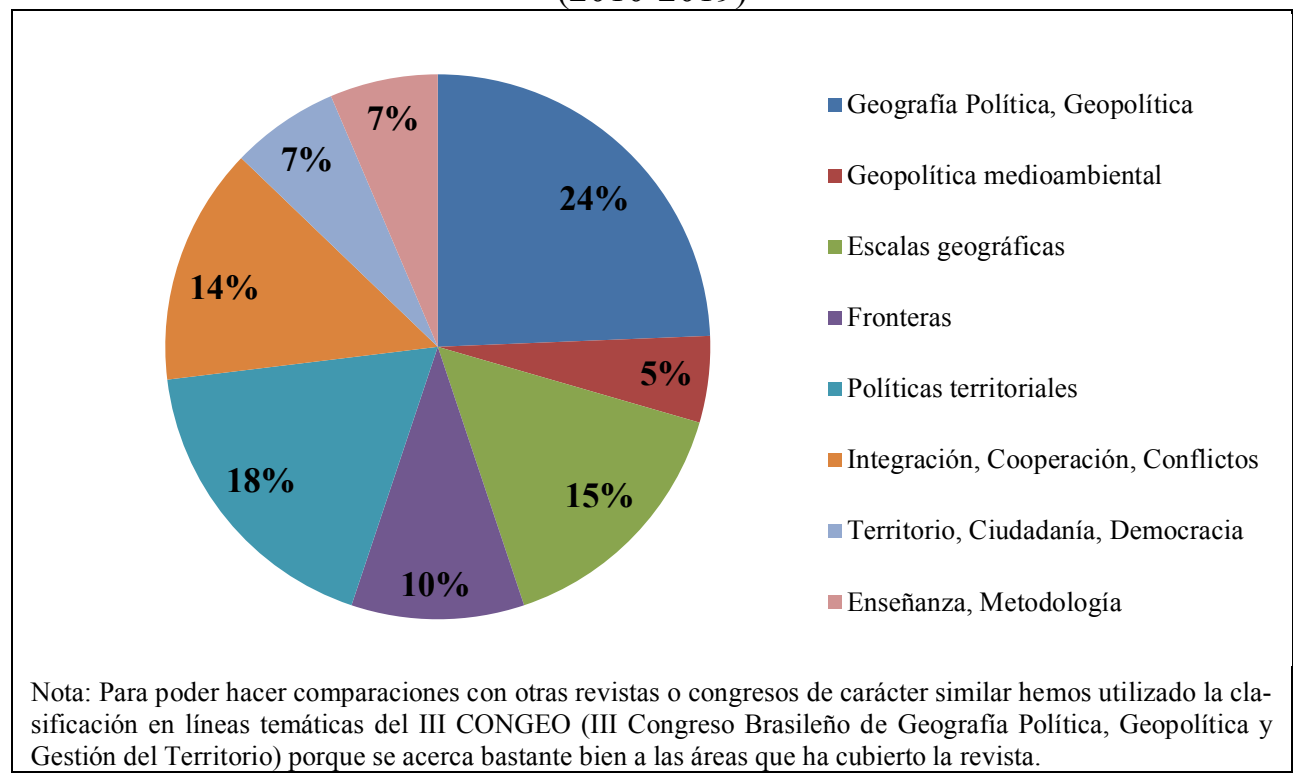

Fuente: Elaborado por Mariano García de las Heras

Las líneas temáticas de los mismos han sido muy diversas, pero siempre relacionadas con el estudio de las relaciones entre espacio y poder. Hemos realizado un análisis a partir de las palabras clave y se ajustan a las siguientes líneas temáticas (Figura 1): en casi todos los números los artículos entran estrictamente dentro de las definiciones de geografía política y geopolítica, que alcanzan un $70 \%$ del total si incorporamos otras líneas muy cercanas como geopolítica medioambiental, escalas geográficas y fronteras; otro buen número de artículos se ha ocupado de políticas territoriales y de cuestiones de integración, cooperación y conflictos; y, finalmente, las líneas de territorio, ciudadanía y democracia, y la de enseñanza y metodología han sumado un $7 \%$ cada una de ellas, lo que supone también una presencia significativa.

Los/as autores/as de todos estos artículos suman un total de 141, lo que significa que un relativamente alto número de los artículos que se han publicado en este pasado decenio se ha escrito en coautoría. En concreto son 30 de 110 - es decir, el $27 \%$ del total - los artículos publicados que tienen dos o más autores, lo que no es inusual en ciencias sociales, donde cada vez más se trabaja en grupo y, por lo tanto, la producción tiende a ser colectiva, menos de lo que ocurre en ciencias naturales y tecnología, pero más que en humanidades. 
Los países de las universidades o instituciones de investigación a las que están adscritos son muy diversos, 21 países en total, pero es cierto que la mayoría se concentran en el área ibérica (31\% entre España y Portugal) y latinoamericana (51\% entre Argentina, Bolivia, Brasil, Chile, Colombia, Costa Rica, Cuba, Ecuador y México) (Figura 2). En todo caso, la distribución es bastante amplia, incluyéndose autores de diversos países europeos y de Estados Unidos, aunque también es cierto que cinco países (Argentina, Brasil, Colombia, España y México) concentran más del $70 \%$ de los autores.

Figura 2. Procedencia de los/as autores/as en Geopolítica(s) (2010-2019) (según país de filiación académica)

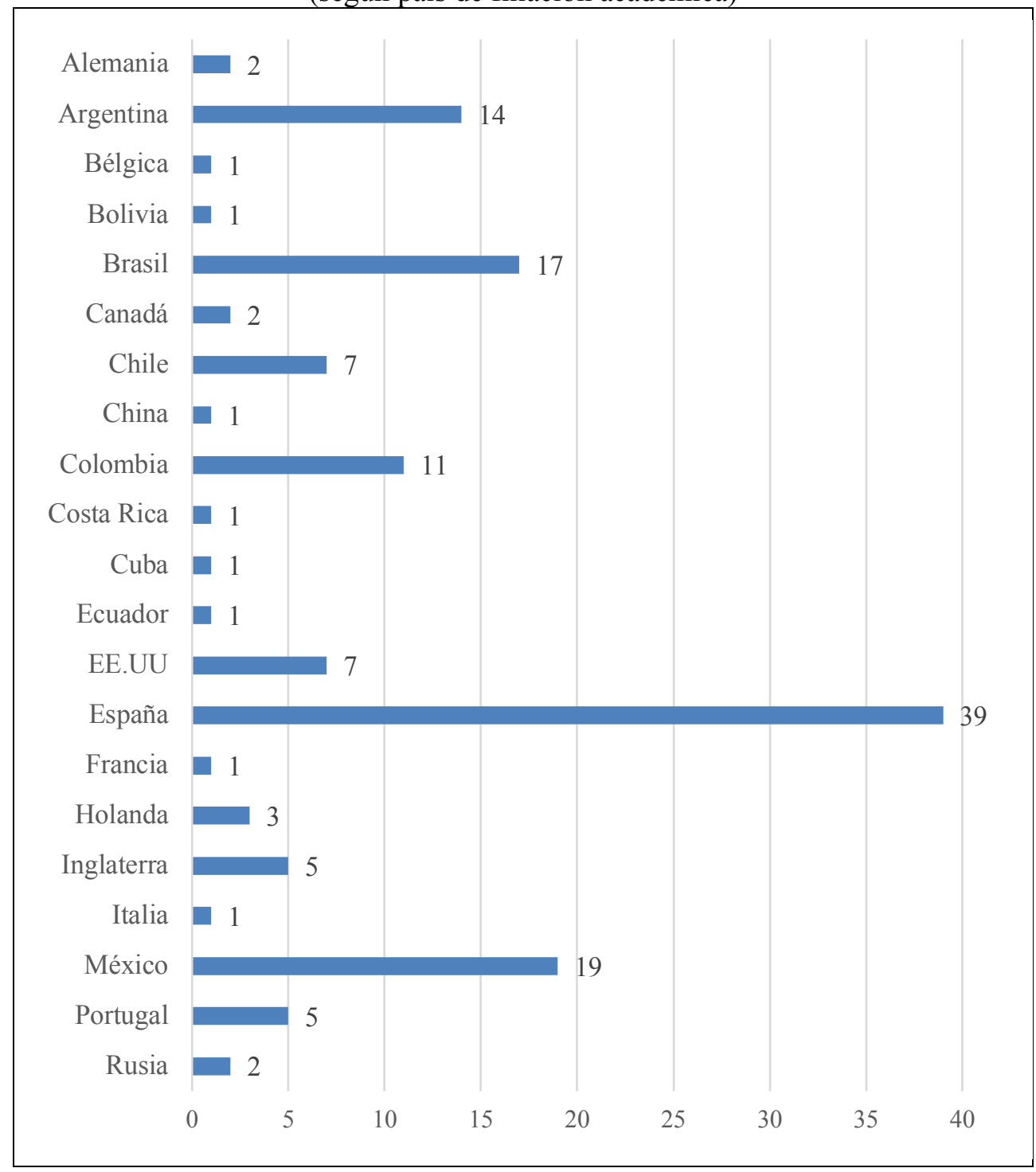

Fuente: Elaborado por Mariano García de las Heras 
En lo referente al género de los autores 110 son varones y 43 son mujeres, lo que significa que hay un desequilibrio de género muy grande, que ha sido especialmente agudo algunos años (Figura 3), como en el 2014, año en el que no tuvimos ninguna autora mujer. Este es un problema importante de la revista cuya magnitud tenemos que intentar, al menos, reducir. Pero que, en todo caso, entendemos en el marco de las desigualdades de género que persisten en las instituciones académicas y universitarias, quizás agravadas, en este caso, por la masculinización/feminización de algunas áreas de investigación.

Figura 3. Autores de artículos de Geopolítica(s) según género (2010-2019)

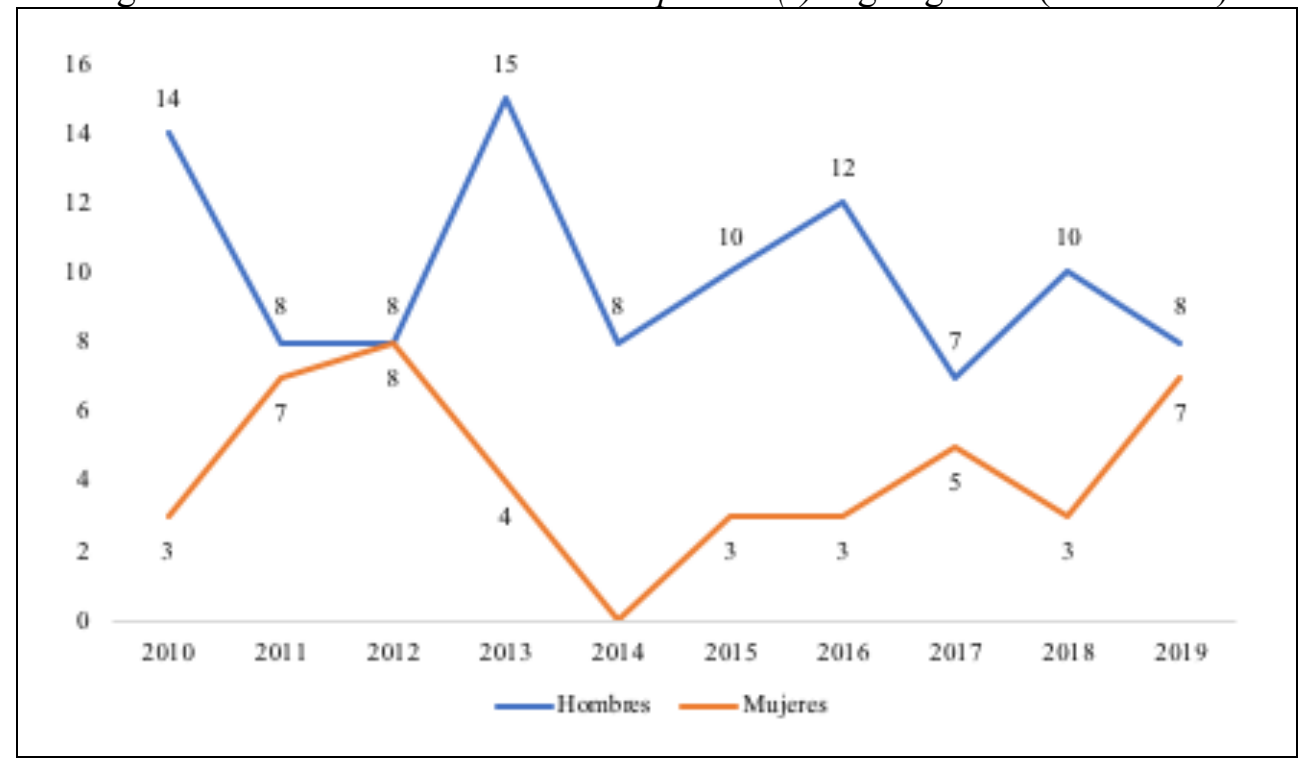

Fuente: Elaborado por Mariano García de las Heras

Dado que somos una revista que publicamos en tres idiomas también es pertinente referirse a los idiomas en que se han publicado los artículos. La mayoría, 87 (79\% del total), se han publicado en castellano, siguiendo los publicados en inglés, 16 ( $15 \%$ del total), y en mucha menor medida en portugués, 7 ( $6 \%$ del total). Creemos que éste es un aceptable nivel de internacionalización lingüística de la revista, especialmente en lo que se refiere al inglés. Pero entendemos que también es conveniente seguir publicando en portugués, ya que el número de artículos procedentes del mundo lusohablante - Brasil en particular - es bastante alto.

La sección de clásicos se ha convertido en una de las que recibe más visitas de la revista, y es, por tanto, uno de nuestros puntos fuertes. Con ella pretendíamos dos cosas: en primer lugar, rescatar textos clásicos, tanto conocidos como olvidados, y hacer una lectura de los mismos desde la actualidad, y, en segundo lugar, permitir a los estudiantes y estudiosos que no dominan otras lenguas - en particular las minoritarias - que accediesen mediante traducciones precisas y especializadas a textos que sino no podrían conocer. 
Las 15 selecciones que se han realizado en la década pasada muestran el carácter eurocéntrico de la geopolítica tradicional: 12 de ellas, el 80\% de las mismas, se produjo en algún país de Europa central y occidental (Alemania, Francia e Inglaterra) o Estados Unidos, mientras que sólo 2 se produjeron en un país de Europa meridional, 1 en Asia y otra en América Latina (Figura 4). Y también es muy fuerte el carácter androcéntrico de la misma, que se refleja en que sólo haya 2 mujeres —una de ellas coautora- entre un universo de 14 autores masculinos.

Figura 4. Autores de la sección de clásicos de Geopolítica(s) según país de procedencia (2010-2019)

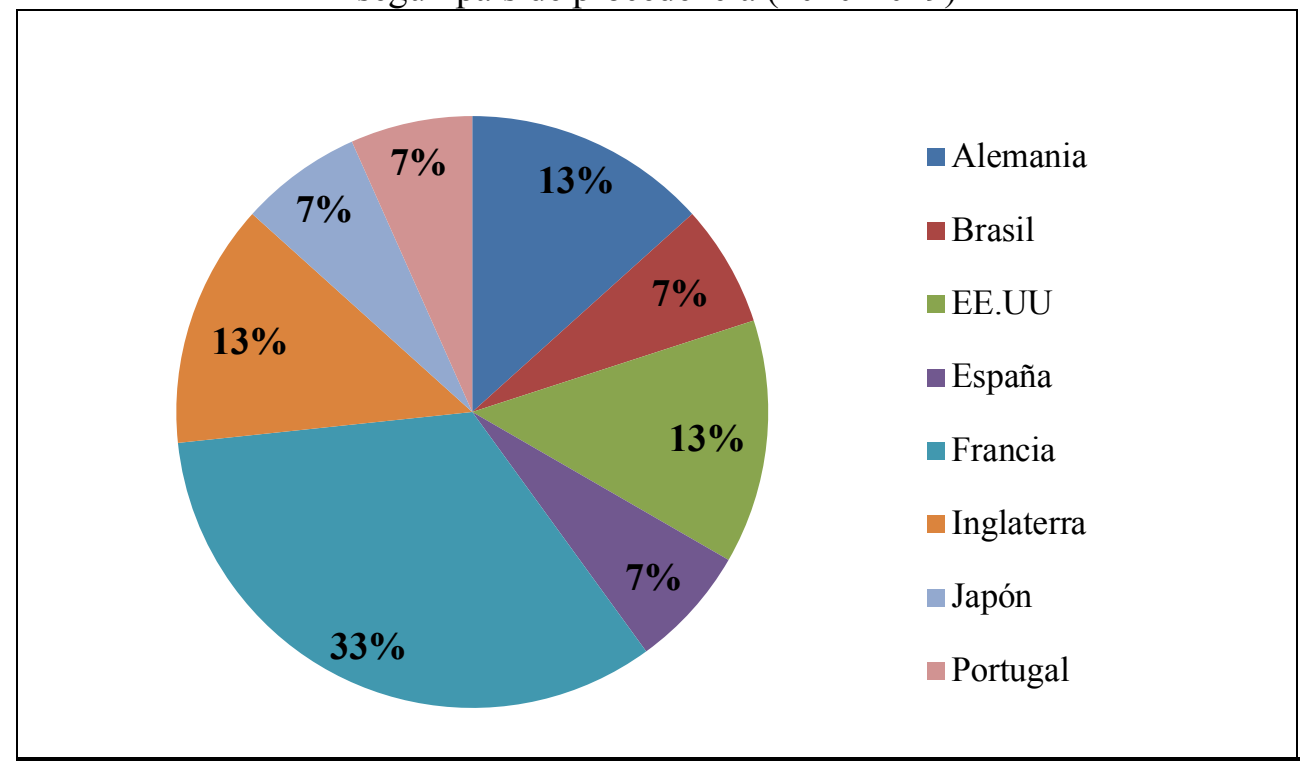

Fuente: Elaborado por Mariano García de las Heras

Sin embargo, la lectura de los clásicos ha sido una lectura fundamentalmente española: 10 de los 15 comentaristas son españoles (67\% del total). Esto podría interpretarse en términos negativos, pero creemos que es consistente con el lugar de edición de la revista, y lejos de ser un hándicap supone un valor añadido, ya que la mayor parte de los análisis sobre los autores clásicos se ha realizado en sus respectivos países y al hacer una lectura desde un lugar alternativo se complejizan más los significados de los textos. Lamentablemente también el desequilibrio de género sigue siendo muy grande entre los autores de los comentarios: 16 varones y sólo 2 mujeres.

En la sección de ensayos bibliográficos se han reseñado un total de 60 obras, a razón de 3 por cada número. Aunque una buena parte de las obras objeto de análisis en la sección ha sido publicada en España (35\%), la procedencia por país, en términos generales, es muy diversa (Figura 5), con textos publicados en catorce países de Europa, América Latina y Norteamérica, sin que ningún país - a excepción del mencionado caso español, con 21 libros - supere en el período analizado un total particular de 6 obras. 
Las obras son tanto de editoriales privadas como públicas, y reflejan las líneas temáticas de interés de la revista, destacando las de geografía política y geopolítica (13 obras) y políticas territoriales (10 obras), y quedando a cierta distancia los trabajos de temáticas de fronteras (7 obras) y otras líneas diversas, que tienen 5 obras o menos. Hay más de autores varones (65) que de mujeres (33), siendo una de ellas de autoría grupal.

Figura 5. Libros de la sección de ensayos bibliográficos de Geopolítica(s) según el lugar de edición de la obra (2010-2019)

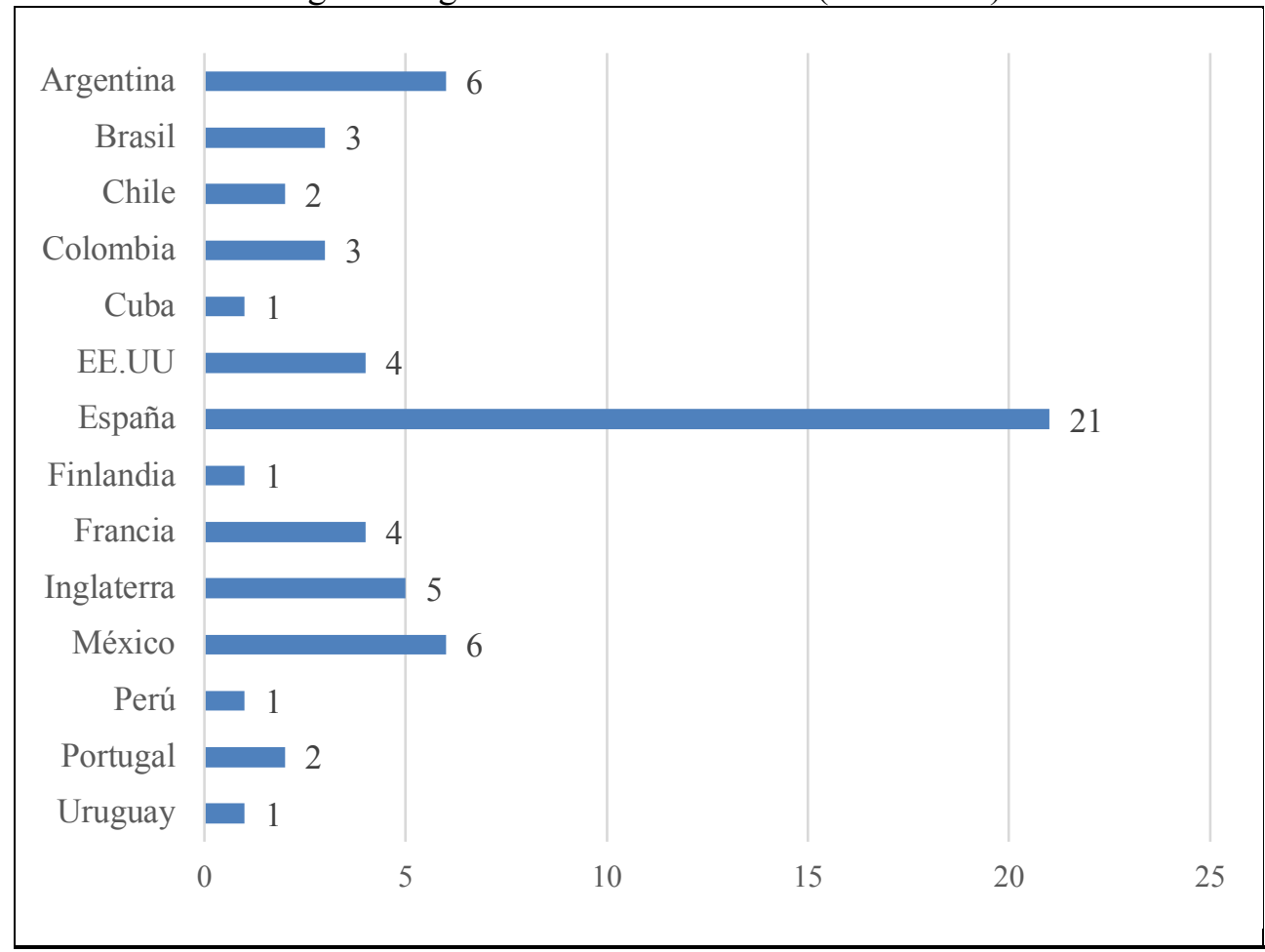

Fuente: Elaborado por Mariano García de las Heras

Los autores de la sección de ensayos bibliográficos son fundamentalmente españoles de filiación académica (54 de 60), lo que es coherente con el hecho de que las reseñas de Geopolítica(s) son encargadas y es frecuente que sean personas de la propia institución o cercanas las que lo realicen. En todo caso es de reseñar que también han participado en la sección autores de filiación académica argentina (1), chilena (1), colombiana (1) y mexicana (2). Pero el desequilibrio de género también existe: han escrito estos ensayos 45 varones y 16 mujeres.

La nueva sección de "Reportes", donde se da información sobre eventos (congresos, seminarios, conferencias, etc.) de geopolítica o disciplinas afines, comenzó en el último número de 2019, por lo que es muy pronto para evaluarla. 


\section{La presencia exterior y la evaluación externa}

La presencia de la revista en las bases de datos especializadas se ha estabilizado, estando en la actualidad incluida en: Dialnet, Emerging Sources Citation Index (ESCI), ÍnDICEs CSIC, International Bibliography of the Social Sciences (IBSS), REDIB (Red Iberoamerica de Innovación y Conocimiento Científico) y Ulrich's Periodicals Directory.

También está presente en los principales índices de evaluación:

- DICE: Es el índice de Difusión y Calidad Editorial de las Revistas Españolas de Humanidades y Ciencias Sociales y Jurídicas, que elabora el Centro de Ciencias Humanas y Sociales (CCHS) del Consejo Superior de Investigaciones Científicas (CSIC) de España. Tiene el problema de que no se actualiza desde 2012. La Valoración de la Difusión Internacional es 1,5, que es Baja. El porcentaje de Internacionalización de las Contribuciones es "Muy Alto", 76,92. La categoría de la revista en la ANEP es B (la tercera de las cuatro categorías de la Agencia).

- ERIH PLUS: European Reference Index for the Humanities and Social Science es un sistema de clasificación de revistas europeas de Ciencias Sociales y Humanidades creado en 2007. Ha sido elaborado hasta 2014 por la European Science Foundation, y desde entonces por la Norwegian Social Science Data Services (NSD). La revista fue incluida en el año 2015 en las categorías de "Human Geography and Urban Studies" y "Political Sciences and International Relations".

- LATINDEX: Es un índice elaborado por un Sistema de Información en Línea para las Revistas Científicas de América Latina, el Caribe, España y Portugal. Se trata de un producto de la cooperación de una red de instituciones que funcionan de manera coordinada para reunir y diseminar información bibliográfica sobre las publicaciones científicas seriadas producidas en la región. Fue incluida en 2010 en el Catálogo 1.0, donde cumplía 35 de 36 características. Está en el Catálogo 2.0 desde 2018 y cumple 36 de las 38 características de éste.

- MIAR: Desarrollada en la Facultad de Biblioteconomía y Documentación de la Universidad de Barcelona y actualizada anualmente, la base de datos Matriz de Información para el Análisis de Revistas reúne información clave para la identificación y el análisis de revistas. El sistema crea una matriz de correspondencia entre las revistas, identificadas por su ISSN y las bases de datos y repertorios que las indizan o incluyen. La revista fue incluida por primera vez en el índice de 2013. El ICDS (Índice compuesto de difusión secundaria) de 2019 es de 7,5, lo que supone que la revista es de "Alta Difusión", que es la segunda categoría del índice.

- Qualis: El índice Qualis-Periódicos fue concebido y refrendado por la Coordenação de Aperfeiçoamento de Pessoal de Nível Superior (CAPES), fundación del Ministerio de Educación de Brasil para la expansión y consolidación del posgrado en el país. Las categorías de clasificación de Qualis (A1, más elevado; A2; B1; B2; B3; B4; B5; C - peso cero) se utilizaron en los procesos de evaluación de los programas de posgrado en lo que se refiere a 
los artículos publicados en revistas científicas. En el período 2013-2016, último disponible, la revista está incluida en las siguientes áreas: "Administração Pública e de Empresas, Ciências Contábeis e Turismo", B3; "Ciência Política e Relações Internacionais", B2; "Geografia", B4; y "Sociologia", B4.

- Scopus: Fue incluida en 2017. Apareció en los índices de 2018, que son los últimos disponibles: el CiteScore 2018 es 0,25 (lo que supone que está en el $3^{\circ}$ cuartil del conjunto de las revistas incluidas en la categoría de "Sociology and Political Science", en concreto la 814 de 1108, en el $3^{\circ}$ cuartil en "Political Science and International Relations", la 357 de 487, y en el $4^{\circ}$ cuartil en "Geography, Planning and Development", la 512 de 628). El Scimago Journal \& Country Rank (SJR) 2018 es 0,106 (que supone estar en el $4^{\circ}$ cuartil en este índice con más ponderaciones que el anterior). Finalmente el Source Normalized Impact per Paper (SNIP) 2018 es 0,120 (este es un factor de medición basado en la comparación de publicaciones dentro de sus campos temáticos, contabilizando la frecuencia con la que los autores/as citan otros documentos y la inmediatez del impacto de la cita).

En las publicaciones es cada vez más importante la altimétrica, es decir, el conjunto de métricas usadas para medir los diferentes impactos de la investigación más allá de las métricas tradicionales de la producción científica. Se trata de una serie de indicadores derivados en muchos casos de herramientas de la web social, que se generan a partir de las interacciones de los usuarios en los espacios web con los materiales generados por los investigadores. La más elemental es el número de descargas de los artículos en la web (Figura 6), que no han dejado de aumentar desde el inicio hasta alcanzar las 68.571 en el año 2019. La revista está incluida en Academia y tiene su propia página en Facebook, con más de 8.000 seguidores/as, y una cuenta de Twitter.

Figura 6. Descargas totales de artículos de Geopolitica(s) (2010-2019)

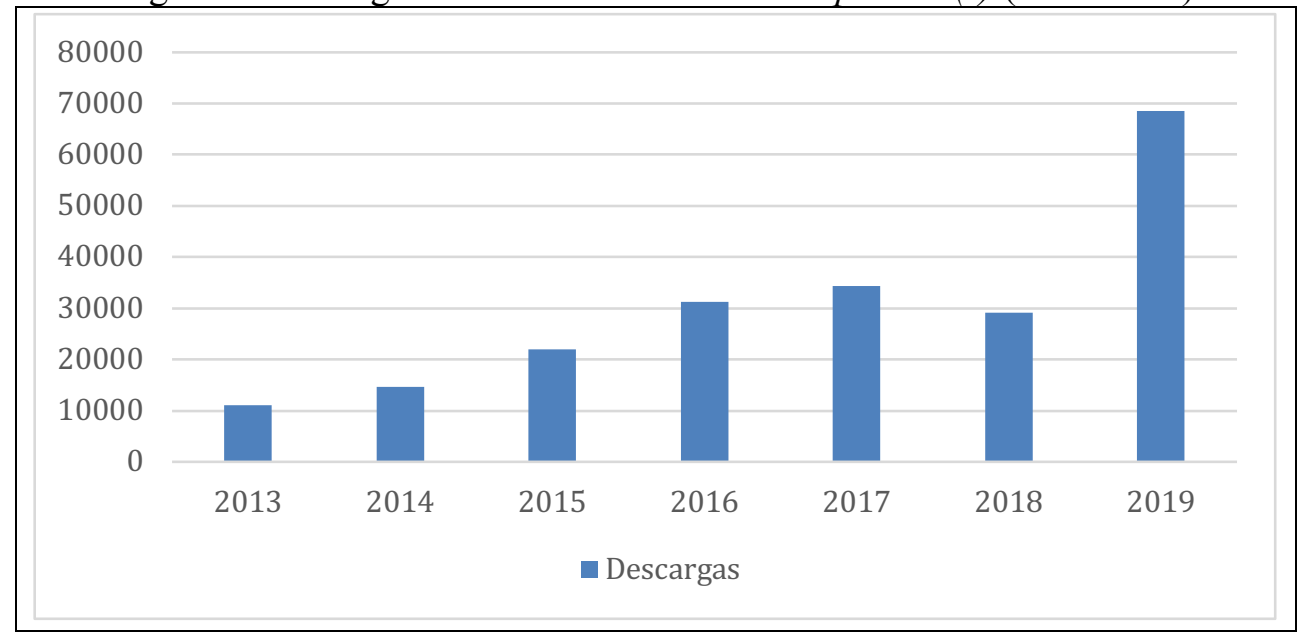

Fuente: Elaboración propia 


\section{El trabajo por delante}

A partir de todos estos datos que acabamos de tratar, y teniendo en cuenta la evolución general de la disciplina - en su sentido más amplio - tenemos que definir las principales líneas de actuación de los próximos años.

En términos globales, la geopolítica, la reflexión sobre el espacio y el poder, se ha asentado definitivamente después del reflorecimiento a finales de los años 1970 al que ya aludíamos en nuestro primer editorial. Ya no sólo es común ver una asignatura de geopolítica en los planes de estudio de grados y másteres en las áreas de geografía o de ciencias políticas y relaciones internacionales, sino que ya abundan más los cursos de posgrado de especialización en nuestros temas. En las asociaciones profesionales de geógrafos y politólogos se han seguido consolidando grupos de investigación especializados. Las revistas punteras de nuestra disciplina (Political Geography, Geopolitics, Hérodote, Society and Space...) no han hecho más que consolidarse en este último decenio. Han mejorado sus posiciones en los índices y se han convertido en plazas de renovación intelectual.

También ha habido una presencia creciente de la geopolítica de orientación clásica en revistas de relaciones internacionales, como Foreign Affairs o Foreign Policy, e incluso en revistas de orientación generalista como Time.

En las áreas regionales en las que más se difunde nuestra revista y de donde vienen la mayoría de nuestros autores es quizás dónde se han producido más cambios en los últimos diez años. La geografía política y la geopolítica tienen ahora mucha mayor presencia tanto en la academia como en los medios. En Brasil existe una Revista de Geopolítica que también comenzó su andadura en 2010, y es previsible que surjan más según se consoliden los núcleos académicos nacionales y regionales activos. En estas circunstancias tenemos que elevar nuestro nivel de exigencia con los artículos, abrir espacios para formulaciones desde la geopolítica crítica sin dejar de publicar trabajos sólidos con otras orientaciones, seguir publicando secciones de clásicos y ensayos bibliográficos que atraigan a los especialistas y estudiosos, $\mathrm{y}$, en definitiva, buscar ser el buque insignia de esa flotilla que esperemos que sea numerosa.

Una mayor calidad de nuestros artículos pasa por mejorar todavía más un proceso de evaluación que ya es minucioso y estricto. Hay que agilizar los procesos para que duren menos, sin que se resienta su rigurosidad.

Las líneas temáticas a las que se adscriben los artículos son ya plurales, a la vez que centradas en el núcleo de la disciplina. Pero es en los autores donde tenemos un déficit importante, no en su procedencia geográfica ni en su adscripción académica, sino, insistimos, en su género. No podemos seguir publicando casi tres veces más artículos de varones que de mujeres. Quizás sea momento de valorar el tomar medidas proactivas de discriminación positiva.

El mismo desequilibrio existe entre los comentaristas de la sección de clásicos y entre los autores de los ensayos bibliográficos, por lo que habrá que hacer un esfuerzo similar al anterior en estos casos también.

Hay algunas bases de datos con fuerte presencia en nuestra área cultural en las que sería conveniente estar: Redalyc, cuyo prestigio y aceptación es creciente en el mundo iberoamericano, y SciELO, que es uno de los índices más reconocidos en América Latina. 
La presencia en los índices evaluadores es importante. No creemos que sea útil rechazarlos. Pero tampoco pueden convertirse en el único criterio a tener en cuenta a la hora de seguir produciendo nuestra revista. Sería bueno mejorar en los índices, entrar en alguno más de los importantes, pero no a cualquier costo. No sin dejar de atender a los autores, no sin evitar presionarles para obtener mejores resultados y más rápidos. Porque no hay que confundir los medios con los fines: el objetivo primordial de una revista académica no es alcanzar una buena clasificación en los índices sino servir a la comunidad de estudiosos y estudiantes de su disciplina. 